Jürgen Troe* and Vladimir G. Ushakov

\title{
Representation of "Broad" Falloff Curves for Dissociation and Recombination Reactions
}

\begin{abstract}
Expressions for representing the pressure dependence of unimolecular dissociation and the reverse recombination reactions are compared. Situations are considered where the broadening of the corresponding falloff curves is particularly pronounced, i.e. where broadening factors at the center of the falloff curves, $F_{\text {cent }}$, are very small and falloff curves correspondingly become very "broad". Such situations arise when unimolecular reactions of molecules with large numbers of low-frequency modes and high-temperature situations are considered. Recombination reactions of polyatomic species in atmospheric chemistry and dissociation reactions of large molecules in combustion are the fields of application of the present results. While previously proposed expressions for asymmetric broadening factors showed artifacts when $F_{\text {cent }}$ decreased to values below about 0.4, alternative functions are described which avoid these problems for situations where $F_{\text {cent }}$ decreases to values far below 0.1 .
\end{abstract}

Keywords: Falloff Curves, Unimolecular Dissociation Reactions, Radical Recombination Reactions, Broadening Factors.

\footnotetext{
*Corresponding Author: Jürgen Troe, Institut für Physikalische Chemie, Universität Göttingen, Tammannstrasse 6, D-37077 Göttingen, Germany, e-mail: shoff@gwdg.de

Jürgen Troe, Vladimir G. Ushakov: Max-Planck-Institut für Biophysikalische Chemie, Am Faßberg 11, D-37077 Göttingen, Germany

Vladimir G. Ushakov: Institute of Problems of Chemical Physics, Russian Academy of Sciences, 142432 Chernogolovka, Russia
}

\section{Introduction}

Thermal dissociation and the reverse recombination reactions in the gas phase are processes of considerable importance in a variety of fields, such as atmospheric chemistry and combustion where they often terminate or initiate the chemical transformation. As collisional activation or deactivation steps participate in these reactions, they show a characteristical pressure dependence. The problem of 
an adequate representation of this pressure dependence has a long history, see e.g. Refs. [1-3]. In a simplistic manner the rate constants $\mathrm{k}$ are approximated in Lindemann-Hinshelwood form as

$$
k / k_{\infty}=F_{L H}(x)=x /(1+x)
$$

where $x=k_{0} / k_{\infty}, k_{\infty}$ denotes the limiting high pressure rate constants, and the pressure dependence enters through the pressure-proportional limiting low pressure rate constants $k_{0}$ (for unimolecular reactions, the $k$ are first-order rate constants, while they are second-order rate constants for recombination). Early unimolecular rate theory like Rice-Ramsperger-Kassel-Marcus (RRKM) theory indicated that falloff curves $k / k_{\infty}$ as a function of the pressure are "broader" than given by Equation (1). One way to represent this phenomenon was the introduction of "broadening" factors $F(x)$ in Equation (1), see Ref. [4], i.e. to extend this equation in the form

$$
k / k_{\infty}=F_{L H}(x) F(x)
$$

where $F(0)=F(+\infty)=1$. The analysis of calculations employing rigid-activated complex, strong-collision RRKM theory led to an approximate representation of $F(x)$ in the form

$$
F(x) \approx F_{\mathrm{cent}}^{1 /\left[1+(\log x / N)^{2}\right]}
$$

with

$$
N \approx 0.75-1.27 \log F_{\text {cent }} .
$$

Accounting for weak collision effects in the collisional energy transfer part of the process, led to an additional broadening [4-6] of the falloff curves, approximately accounted for by a modification of the "center broadening factor" $F_{\text {cent }}$. Equations (1)-(4) proved useful in practical representations of dissociation and recombination rate constants [7-9] as long as $F_{\text {cent }}$ was not too small, e.g., being between about 0.4 and unity.

Problems with Equations (1)-(4) arose when $F_{\text {cent }}$ assumed values below about 0.4 . The broadening factors then become asymmetric, i.e. $F(x)$ differs from $F(1 / x)$ in Equation (2), and the minimum of $F(x)$ moves away from $x=x_{\text {cent }}=1$. The use of Equations (1)-(4) then even introduces "wiggles" of the falloff curve (i.e. inflection points of $k$ as a function of the pressure) which clearly are artifacts of the approximation of $F(x)$ by Equation (3). Alternative expressions thus are required for situations where $F_{\text {cent }}$ falls to very small values. This will happen when the system consists of large numbers of oscillators, when their fre- 
quencies are low, and when the temperatures are high. A first glance on the order of magnitude of $F_{\text {cent }}$ to be expected was given by classical Kassel theory [2] where $F_{\text {cent }}$ was related to the number of oscillators $s$ and the bond energy $E_{0}$ of the dissociating molecules. Representations of $F(x)$ in terms of the corresponding classical "Kassel integrals" were given in Ref. [3]. Introducing quantization of the oscillators, effective values $s_{\text {eff }}$ of the numbers of oscillators generally were smaller than $\mathrm{s}$ and $F_{\text {cent }}$ differed from the values given by classical Kassel theory. Convenient ways to account for this were proposed in Ref. [5] and expressions of the type of Equations (2)-(4) were developed. It was, however, shown [10] that there cannot be a unique form of $F(x)$ which is adequate for all reactions. Instead each reaction has its specific form of $F(x)$. For systems with the same $F_{\text {cent }}$, nevertheless, $F(x)$ does not deviate too much from an average general form [10].

Although Equations (1)-(4) have found wide application and are useful in many situations, their evident problems have stimulated search for better approximations, either for the broadening factors $F(x)$ or, abandoning the separation in Equation (2), of $k / k_{\infty}$ as a more general function of $k_{0} / k_{\infty}$. A number of expressions have been proposed, see e.g. [10-16]. As there is no unique form of the broadening factors $F(x)$, one cannot claim that the one expression is better than the other in all generality. Instead, realistic form, simplicity, and flexibility of the expressions have to be weighted against each other. At the same time, improvements of the results have to be weighted against the need to change an established format like Equation (3) (or even the simpler policy of using Equations (1)-(4) with fixed $F_{\text {cent }}=0.6$ such as used in [9]).

In our recent work [17] on the dissociation-recombination reaction system $\mathrm{C}_{2} \mathrm{~F}_{4} \Leftrightarrow 2 \mathrm{CF}_{2}$ we encountered the situation that $F_{\text {cent }}$ decreases to values near 0.1. Modeling falloff curves with earlier, previously proposed model functions for $F(x)$ here led to the described unsatisfactory appearance of wiggles. In addition, different model functions for $F(x)$ lead to different limiting rate constants $k_{0}$ and $k_{\infty}$ when fitted to experimental parts of the falloff curve. It, therefore, appeared desirable to test alternative expressions for $F(x)$ with respect to their performance in situations when $F_{\text {cent }}$ is smaller than about 0.4. Besides simplified methods to predict $F_{\text {cent }}$ such as suggested in $[5,18]$, one also needs information about the uncertainties of $k_{0}$ and $k_{\infty}$ obtained by fitting $F(x)$ to limited parts of experimental falloff curves. This is the issue of the present article. We test the suitability of various functional forms of $F(x)$ for application to low $F_{\text {cent }}$ cases. We use data for the dissociations of $\mathrm{H}_{2} \mathrm{CO}$ [10] and $\mathrm{C}_{2} \mathrm{~F}_{4}$ [17] as specific examples, but we also extend our treatments to model situations where $F_{\text {cent }}$ falls to values much smaller than 0.1 . 


\section{Fitting of experimental falloff curves}

Equations (1)-(4) were derived from RRKM simulations of rigid-activated complex unimolecular reactions $[4,5]$. Under conditions where the effective number of oscillators $s_{\text {eff }}$ was large and the broadening became very pronounced, the falloff curves increasingly showed asymmetries (i.e., $F(x) \neq F(1 / x)$ ). In addition, the minima of $F(x)$ moved to values of $x<1$. Such effects were also found to gain importance when rotational effects were taken into account $[10,18]$ such as this is the case for unimolecular bond fission and the reverse radical recombination reactions. A variety of expressions were proposed to approximate the resulting broadening factors, such as

$$
F(x) \approx F_{\text {cent }}^{1 /\left\{1+[|\log (1.4 x)| /(N+\Delta N)]^{2}\right\}}
$$

with $\Delta N=0.3 \log F_{\text {cent }}$ for $\log (1.4 x)>0$ and $\Delta N=-0.7 \log F_{\text {cent }}$ for $\log (1.4 x)<0$ from Ref. [10] (being close to the propositions of Refs. [4, 5]), or

$$
F(x)=1-\left(1-F_{\text {cent }}\right) \exp \left\{-[\log (1.5 x) / N]^{2} / N^{*}\right\}
$$

with $N^{*}=2$ for $\log (1.5 x)>0$ and $N^{*}=2[1-0.15 \log (1.5 x)]$ for $\log (1.5 x)<0$ from Ref. [10]. In the present work we test alternative expressions in the form

$$
F(x)=\left(1+x / x_{0}\right) /\left[1+\left(x / x_{0}\right)^{n}\right]^{1 / n}
$$

with

$$
n=\left[\ln 2 / \ln \left(2 / F_{\text {cent }}\right)\right]\left[1-b+b\left(x / x_{0}\right)^{q}\right]
$$

where $q=\left(F_{\text {cent }}-1\right) / \ln \left(F_{\text {cent }} / 10\right)$, the parameter $x_{0}$ being in the range 0.9-1.1 (mostly near to 1 , see below) and $b$ in the range 0.1-0.25 (mostly near to 0.2, see below). As mentioned above detailed calculations of falloff curves for a large series of model reactions in Ref. [10] showed that broadening factors of falloff curves with the same $F_{\text {cent }}$ do not fall onto one curve but into a band with about $\pm 10 \%$ deviation around an average curve of $F(x)$ to be modeled by expressions like Equations (3), (6)-(8). The range of the parameters $x_{0}$ and $\mathrm{b}$ and their average values as given above reflect this band of calculated $F(x)$ ). It was found that Equation (6) well represented the asymmetries of the broadening factors $F(x)$ as long as $F_{\text {cent }}>0.4$. However, the application of Equation (6) to the thermal dissociation of $\mathrm{C}_{2} \mathrm{~F}_{4}$ in Ref. [17], for which $F_{\text {cent }} \approx 0.1$, showed that the modeled complete falloff curves also showed the mentioned wiggles which are clearly artifacts of the chosen expression. Figure 1 illustrates this observation. In this example both $k_{\infty}$ and $F_{\text {cent }}$ were fixed to model calculations and $k_{0}$ was used as a free parameter 


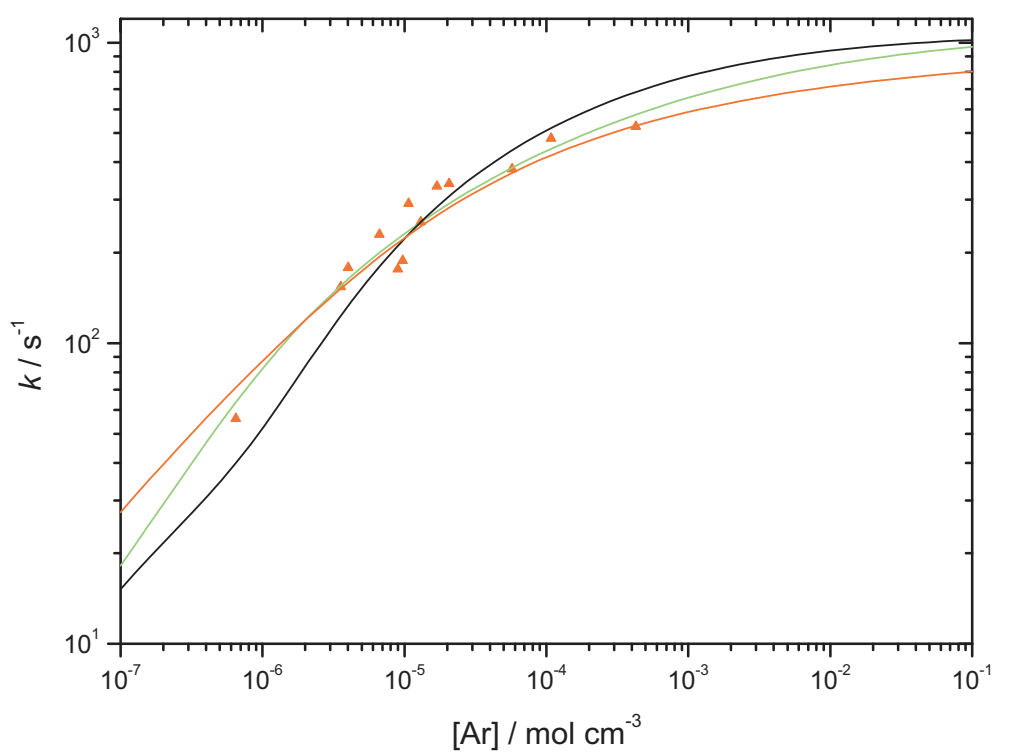

Figure 1: Falloff curves for the dissociation reaction $\mathrm{C}_{2} \mathrm{~F}_{4}(+\mathrm{Ar}) \rightarrow 2 \mathrm{CF}_{2}(+\mathrm{Ar})$ at $1200 \mathrm{~K}$ (symbols: experimental data from [17]; fits with fixed $k_{\infty}=1.06 \cdot 10^{3} \mathrm{~s}^{-1}$ and $F_{\text {cent }}=0.1$ and Equations (1)-(4): red curve leading to $k_{0}=[\mathrm{Ar}] 3 \cdot 10^{9} \mathrm{~cm}^{3} \mathrm{~mol}^{-1} \mathrm{~s}^{-1}$; fits with Equation (6): black curve leading to $k_{0}=[\mathrm{Ar}] 9.8 \cdot 10^{8} \mathrm{~cm}^{3} \mathrm{~mol}^{-1} \mathrm{~s}^{-1}$; fits with Equations (7) and (8) with $x_{0}=1$ and $b=0.2$ : green curve leading to $k_{0}=[\mathrm{Ar}] 1.8 \cdot 10^{9} \mathrm{~cm}^{3} \mathrm{~mol}^{-1} \mathrm{~s}^{-1}$ ).

for a mean-least squares fit to the experiments. One notices that the falloff curve with the symmetric broadening factor of Equations (1)-(4) exaggerates the deviation of $k$ from $k_{\infty}$, while the asymmetric broadening factor of Equation (6) avoids this problem but introduces wiggles. Our new representation of the broadening factor by Equations (7) and (8) provides a much better fit to the experimental data from Refs. [17] and [19]. Fixing $k_{\infty}$ and $F_{\text {cent }}$ to modeled values in this case leads to quite different values of the fitted $k_{0}$ which varies by a factor of three for the three representations.

Using the experimental data (see Ref. [17]) alone and allowing $k_{0}, k_{\infty}$, and $F_{\text {cent }}$ all to be optimized, markedly changes the situation such as illustrated in Figure 2. Fitted values of $F_{\text {cent }}$ move up from 0.1 to the range $0.33-0.44$, the fitted $k_{\infty}$ decreases from $1.1 \cdot 10^{3} \mathrm{~s}^{-1}$ to about $0.6 \cdot 10^{3} \mathrm{~s}^{-1}$, while the spread of $k_{0}$ is reduced from a factor of 3 to about a factor of 1.7. As a consequence of increasing $F_{\text {cent }}$, the wiggles from Equation (6) nearly disappear and also the asymmetries from Equations (6)-(8) loose importance. On the basis of the experimental points alone no reliable fit of the three characteristic parameters $k_{0}, k_{\infty}$, and $F_{\text {cent }}$ would have been possible. Even if experimental data over much larger pressure ranges 


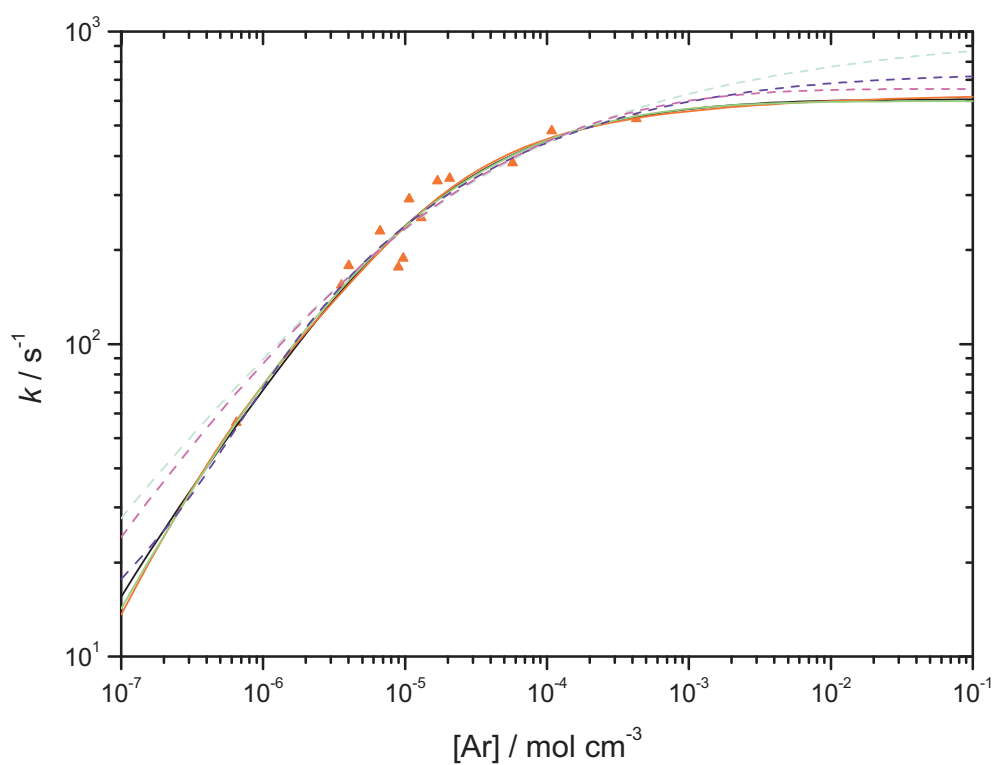

Figure 2: As Figure 1 (symbols: experimental data from [17]; full curves: fits with variable $k_{0}, k_{\infty}$, and $F_{\text {cent }}$; red curve: Equations (1)-(4) leading to $k_{0}=[\mathrm{Ar}] 1.9 \cdot 10^{8} \mathrm{~cm}^{3} \mathrm{~mol}^{-1} \mathrm{~s}^{-1}$, $k_{\infty}=6.6 \cdot 10^{2} \mathrm{~s}^{-1}$, and $F_{\text {cent }}=0.44$, black curve: Equation (6) leading to $k_{0}=[\mathrm{Ar}]$ $3.3 \cdot 10^{8} \mathrm{~cm}^{3} \mathrm{~mol}^{-1} \mathrm{~s}^{-1}, k_{\infty}=6.1 \cdot 10^{2} \mathrm{~s}^{-1}$, and $F_{\text {cent }}=0.33$, green curve: Equations (7) and (8) leading to $k_{0}=[\mathrm{Ar}] 2.7 \cdot 10^{8} \mathrm{~cm}^{3} \mathrm{~mol}^{-1} \mathrm{~s}^{-1}, k_{\mathrm{\infty}}=6.0 \cdot 10^{2} \mathrm{~s}^{-1}$, and $F_{\text {cent }}=0.39$; dashed curves: fits with fixed $F_{\text {cent }}=0.1$; light blue curve: Equations (1)-(4) leading to $k_{0}=[\operatorname{Ar}] 2.8 \cdot 10^{9} \mathrm{~cm}^{3} \mathrm{~mol}^{-1} \mathrm{~s}^{-1}$ and $k_{\infty}=1.2 \cdot 10^{3} \mathrm{~s}^{-1}$, dark blue curve: Equation (6) leading to $k_{0}=[\mathrm{Ar}] 1.9 \cdot 10^{9} \mathrm{~cm}^{3} \mathrm{~mol}^{-1} \mathrm{~s}^{-1}$ and $k_{\infty}=7.3 \cdot 10^{2} \mathrm{~s}^{-1}$, magenta curve: Equations (7) and (8) leading to $k_{0}=[\mathrm{Ar}] 3.9 \cdot 10^{9} \mathrm{~cm}^{3} \mathrm{~mol}^{-1} \mathrm{~s}^{-1}$ and $k_{\infty}=6.6 \cdot 10^{2} \mathrm{~s}^{-1}$ ).

would be available (see e.g. the falloff curve for $\mathrm{OH}+\mathrm{NO}_{2}(+M) \rightarrow \mathrm{HONO}_{2}(+M)$ with variations of $[M]$ over 6 orders of magnitude, such as analyzed in Ref. [20]), unique fits for $k_{0}, k_{\infty}$, and $F_{\text {cent }}$ would not be feasible. In this case theoretical modeling of at least one of the three quantities would be necessary. Figure 1 demonstrated the results when two parameters $\left(k_{\infty}\right.$ and $\left.F_{\text {cent }}\right)$ were fixed to theoretical estimates. Figure 2, besides fits with three fit parameters, includes fits with fixed $F_{\text {cent }}=0.1$. In this case considerable variations of the fitted $k_{0}$ and $k_{\infty}$ are obtained. Also the wiggles of a fit with Equation (6) again become pronounced at the low pressure end of the falloff curve.

The shown example of a fit of various falloff expressions to a limited set of experimental data thus shows that unique results can only be obtained when theoretical modeling of some of the characteristic parameters $k_{0}, k_{\infty}$, and $F_{\text {cent }}$ can be made. In addition, one needs to explore which of the expressions under dis- 


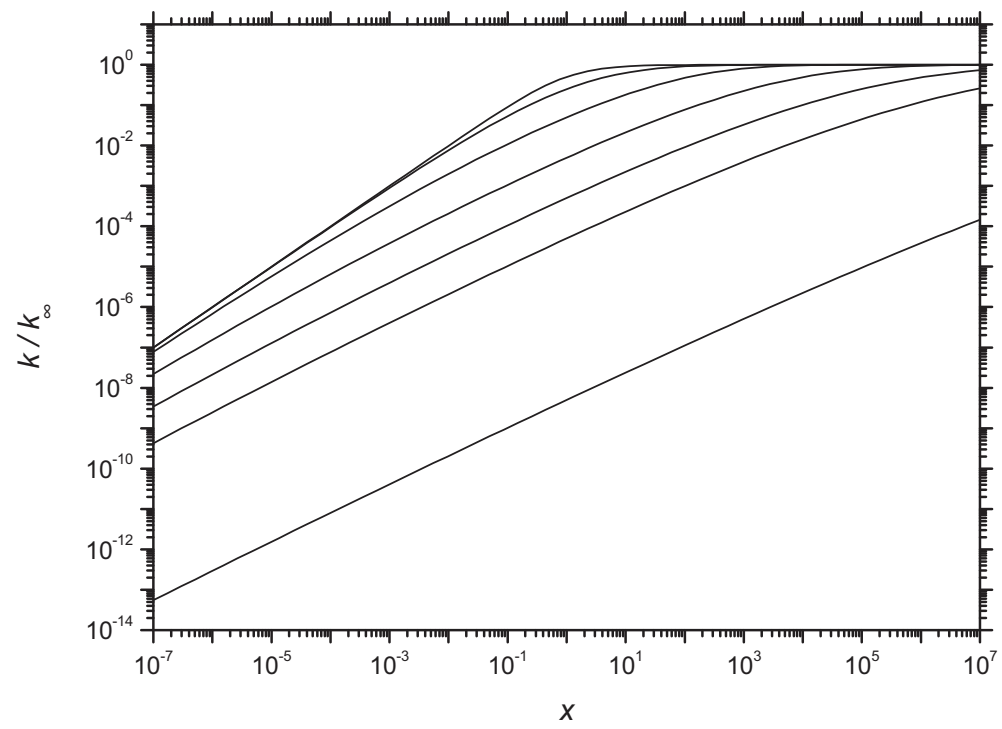

Figure 3: Falloff curves from Equations (7) and (8) (with $x_{0}=1$ and $b=0.2$; curves from top to bottom with $F_{\text {cent }}=1,0.5,10^{-1}, 10^{-2}, 10^{-3}, 10^{-4}, 10^{-8}$ ).

cussion fits best complete theoretical falloff curves within the spread of possible results demonstrated in Ref. [10]. The latter is addressed in the following section.

\section{Asymmetric broadening factors for "broad" falloff curves}

In this section we explore the performance of the broadening factor of Equations (7) and (8) with respect to the appearance of wiggles and in application to specific theoretical falloff curves. First, we show that Equations (7) and (8) avoid the appearance of wiggles even when $F_{\text {cent }}$ decreases to values $F_{\text {cent }} \ll 1$, i.e. when the falloff curves become extremely broad. Figure 3 shows a set of falloff curves from Equations (7) and (8) with $F_{\text {cent }}$ between $10^{-8}$ and unity. The wiggles of Equation (6) have disappeared while the asymmetric character of the curves increasingly becomes visible for decreasing $F_{\text {cent }}$. Although values of $F_{\text {cent }}$ as low as $10^{-8}$ are not relevant in practice, it is important to have a general representation of broadening factors which does not show wiggles when $F_{\text {cent }}$ falls below about 0.4.

Next, we compare the broadening factors of Equations (7) and (8) with theoretically modeled results such as derived in Ref. [10]. We have chosen two ex- 


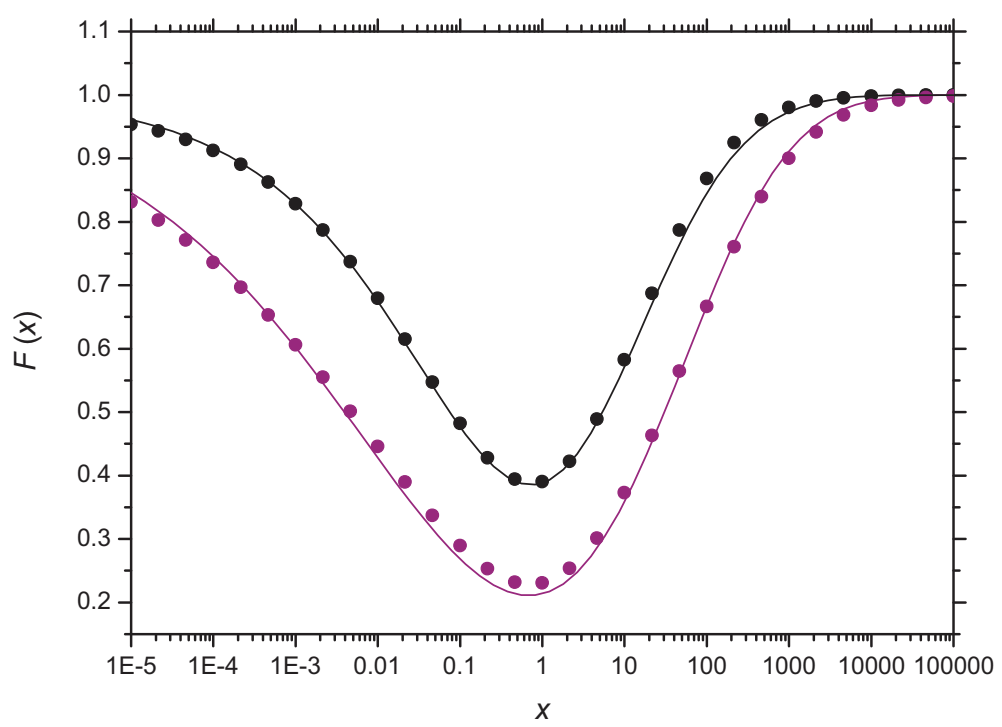

Figure 4: Comparison of fitted broadening factors $F(x)$ employing Equations (7) and (8) (lines) with theoretical modeling results (points). (Upper curve for weak-collision two-channel dissociation of formaldehyde at $1000 \mathrm{~K}$ : calculated points from [10]; lower curve: bond fission of an artificial 8-atomic molecule leading into two spherical top fragments under weak collisions conditions: calculated points from [10], see text).

amples which account for a series of complicated details. As one example we have taken results for the total rate constant of the two-channel dissociation of formaldehyde at $1000 \mathrm{~K}$, including contributions from loose, roaming, and rigid activated complexes. In addition, for this example, we have included weak collision effects (such as documented in Figure 3 of Ref. [10] from where the lowest $F(x)$ was selected). The upper of the two curves in Figure 4 corresponds to this example. The figure compares the theoretical results (shown as points) with a fit by Equations (7) and (8). The agreement is very good (with the parameters $x_{0}$ and $b$ taken as the standard values of $x_{0}=1$ and $b=0.15$ ). In this case $F_{\text {cent }}$ is the only fit parameter for $F(x)$ (a value of $F_{\text {cent }}=0.39$ here was fitted). The asymmetry of the theoretical $F(x)$ is well reproduced. As a second example we have taken theoretical results for an artificial molecular system consisting of an eightatomic molecule dissociating by simple bond fission into two spherical tops in an isotropic potential; again, weak collision effects were included (such as documented in Figure 8 of Ref. [10] from where the lowest calculated $F(x)$, at a given $x$, were selected). The lower of the two curves in Figure 4 illustrates the fit of Equations (7) and (8) with the same $x_{0}=1$ and $b=0.15$ to this example. Again the 
fit gives very good results, well reproducing the asymmetry of $F(x)$ for a case where the falloff curve is already very broad (a value of $F_{\text {cent }}=0.22$ here was fitted).

\section{Discussion}

We have illustrated in Sections 2 and 3 that Equations (7) and (8) very well reproduce asymmetric broadening factors for "broad" falloff curves, as characterized by values of the center broadening factors $F_{\text {cent }}$ below about 0.4. Although in principle Equations ( 7 ) and (8) contain the three fit parameters $F_{\text {cent }}, x_{0}$, and $b, x_{0}$ and $b$ can be fixed as standard values of $x_{0}=1$ and $b=0.15$. Falloff curves then again would be characterized by three parameters only, i.e. by $k_{0}, k_{\infty}$, and $F_{\text {cent }}$. Previously proposed expressions for $F(x)$ like Equations (1)-(4) or Equations (5) and (6) work equally well for $F_{\text {cent }}>0.4$, although not providing unique sets of the three parameters when allowed to vary freely in fitting. The new expressions of Equations (7) and (8) allow for the representation of falloff curves over much larger ranges of $F_{\text {cent }}$, covering all cases of practical need.

One should finally note that polynomial expressions for $F(x)$ have also been suggested before, e.g. in Ref. [12] where

$$
k=\left[k_{0}^{a}+k_{\infty}^{a}\right]^{1 / a}
$$

was employed. For $a=-1, F(x)=1$ in Equations (1) and (2) is obtained. However, the recommended expressions for the parameter a (see Equation (26) of Ref. [12]) were found to be much less suitable to account for the asymmetries of $F(x)$ and the link between the magnitude of $F_{\text {cent }}$ and the extent of asymmetry and the width of $F(x)$.

In conclusion we state that Equations (7) and (8) provide a satisfactory representation of broadening factors $F(x)$ for "broad" falloff curves with center broadening factors $F_{\text {cent }}$ being below about 0.4 . At values of $F_{\text {cent }}$ above 0.4, asymmetries of $F(x)$ are only of minor importance and expressions like Equations (1)(6) equally well can be used to fit falloff curves. However, different fitted value of $k_{0}, k_{\mathrm{\infty}}$, and $F_{\text {cent }}$ are to be expected when different expressions are used. For $F_{\text {cent }}<0.4$, artifacts arise when Equations (1)-(6) are used, such that Equations (7) and (8) become the representation of choice. Fixing $x_{0}=1$ and $b=0.15$, the falloff representation remains a three-parameter approach (using $k_{0}, k_{\infty}$, and $F_{\text {cent }}$ ). Nevertheless, one should keep in mind that deviations of about $\pm 10 \%$ around Equations (7) and (8) are possible for specific reaction systems [10]. 
Received October 18, 2013; accepted November 27, 2013.

\section{References}

1. T. Baer and W. L. Hase, Unimolecular Reaction Dynamics. Theory and Experiments, Oxford University Press, New York (1996).

2. L. S. Kassel, Kinetics of Homogeneous Gas Phase Reactions, Chemical Catalog Co., New York (1932).

3. J. Troe, Ber. Bunsenges. Phys. Chem. 78 (1974) 478.

4. J. Troe, J. Phys. Chem. 83 (1979) 114.

5. J. Troe, Ber. Bunsenges. Phys. Chem. 87 (1983) 161.

6. R. G. Gilbert, K. Luther, and J. Troe, Ber. Bunsenges. Phys. Chem. 87 (1983) 169.

7. D. L. Baulch, C. T. Bowman, C. J. Cobos, R. A. Cox, T. Just, J. A. Kerr, M. J. Pillling, D. Stocker, J. Troe, W. Tsang, R. W. Walker, and J. Warnatz, J. Phys. Chem. Ref. Data 34 (2005) 757.

8. R. Atkinson, D. L. Baulch, R. A. Cox, J. N. Crowley, R. F. Hampson, R. G. Hynes, M. E. Jenkin, M. J. Rossi, J. Troe, and T. J. Wallington, Atmos. Chem. Phys. 8 (2008) 4141.

9. S. P. Sander, R. R. Friedl, J. R. Barker, D. M. Golden, M. J. Kurylo, P. H. Wine, J. P. D. Abbott, J. B. Burkholder, C. E. Kolb, G. K. Moortgat, R. E. Huie, and V. L. Orkin, JPL Publication 10-6 (June 10, 2011).

10. J. Troe and V. G. Ushakov, J. Chem. Phys. 135 (2011) 054304.

11. Z. Pawlowska, W. C. Gardiner, and I. Oref, J. Phys. Chem. 97 (1993) 5024.

12. A. Kazakov, H. Wang, and M. Frenklach, J. Phys. Chem. 98 (1994) 10598.

13. O. Prezhdo, J. Phys. Chem. 99 (1995) 8633.

14. P. K. Venkatesh, J. Phys. Chem. A 104 (2000) 280.

15. C. J. Cobos and J. Troe, Z. Phys. Chem. 217 (2003) 1031.

16. P. Zhang and C. K. Law, Int. J. Chem. Kinet. 43 (2011) 31.

17. C. J. Cobos, A. Croce, K. Luther, L. Soelter, E. Tellbach, and J. Troe, J. Phys. Chem. A 117 (2013) 11420.

18. J. Troe and V. G. Ushakov, Faraday Discuss. 119 (2001) 145.

19. K. P. Schug and H. Gg. Wagner, Ber. Bunsenges. Phys. Chem. 82 (1978) 719.

20. J. Troe, J. Phys. Chem. A 116 (2012) 6387. 